\title{
Effect of friction on the interface crack loaded in shear
}

\author{
MARIA COMNINOU
}

The University of Michigan, Ann Arbor, Michigan 48109, USA

and

\section{J. DUNDURS}

Northwestern University, Evanston, Illinois 60201, USA

(Received March 8, 1979)

\begin{abstract}
The conventional formulation used in the past for problems involving interface cracks leads to a physical contradiction: The two sides of the crack are assumed to be free of tractions in the formulation, but the crack faces are seen to overlap after the solution is constructed. This unsatisfactory feature can be eliminated by introducing contact zones at the tips of an interface crack. The present article investigates the effect of friction in the contact zones for loads that start from zero and are increased monotonically. As an application, shear loading is considered, and the problem is reduced to a singular integral equation with a Cauchy-type kernel which is solved numerically. The results show that one of the contact zones is large and that friction affects the global nature of the stress fields. The results worked out include also the stress intensity factors, crack opening displacement, and the pressure distribution in the larger contact zone.
\end{abstract}

\section{RÉSUMÉ}

La formulation habituelle utilisée dans le passé pour des problèmes de fissures interfaciales conduit à une contradiction physique: Les deux lèvres de la fissure doivent être libres de contraintes, mais la solution peut impliquer une interpénétration des bords. Il est possible d'éliminer cette contradiction physique en introduisant des zones de contact aux pointes de la fissure. Le présent article étudie l'effet du frottement dans les zones de contact pour des charges croissantes à partir de zero. Comme application, on considère une charge de cisaillement et le problème se réduit à une équation intégrale singulière avec un noyau de type Cauchy. Cette équation est résolue numériquement. Les résultats montrent que l'une des zones de contact est grande et que le frottement modifie l'ensemble du champ de contraintes. On a aussi obtenu les facteurs d'intensité de contraintes, l'ouverture de la fissure et la distribution du pression dans la plus grande zone de contact.

\section{Introduction}

A large number of elasticity solutions for interface cracks are based on the assumption that the transition from adhesion to separation is direct. This assumption leads to oscillatory singularities and to overlapping of the crack faces $[1,2]$. The 
interpenetration of material means that no direct transition from adhesion to separation is generally possible, and suggests that there must be intervening contact zones at the tips of an interface crack. It has been confirmed from the appropriate asymptotic analysis and the solution of some specific problems that it is indeed possible to avoid the previously encountered contradictions by allowing contact zones at the crack tips [3-5].

In the present article, we continue the study of the interface crack with contact zones by including the effect of friction. Elasticity problems involving friction are difficult because the stresses depend not only on the instantaneous magnitudes of the applied forces, but also involve the history of loading, and such problems generally require an incremental formulation [6]. No incremental formulation is necessary for the interface crack, however, if the loading is started from zero and increased monotonically. The reason for this simplification is that slip takes place over the whole extent of the contact zones, and that the lengths of the contact zones do not depend on the level reached by the monotonically increased loads. The assertion made here follows from some recently obtained general results for receding contacts with friction [7]. Our considerations in this article are restricted to loads of the nature mentioned.

\section{Formulation}

Consider the interface crack between the two solids with the shear moduli $\mu_{1}, \mu_{2}$ and Poisson's ratios $\nu_{1}, \nu_{2}$, as indicated in Figure 1. The length of the crack is $2 L$. Under the action of the applied loads, the crack opens in the interval $(-a, b)$, but its two faces remain in contact in the intervals $(-L,-a)$ and $(b, L)$. The constants $a$ and $b$ defining the extent of the contact zones are unknowns to be determined in the course of solution.

The boundary conditions in the open part $-a<x<b$ of the crack are

$$
\begin{aligned}
& \sigma_{x y}(x, 0)=\sigma_{y y}(x, 0)=0 \\
& g(x)=u_{y}^{(2)}(x, 0)-u_{y}^{(1)}(x, 0) \geq 0
\end{aligned}
$$

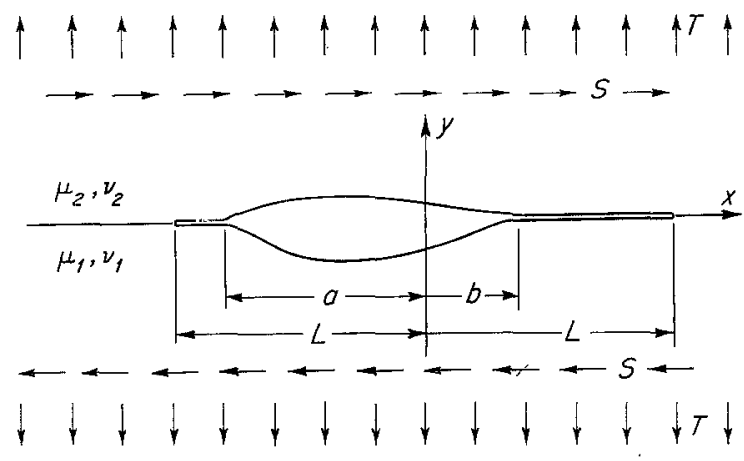

Figure 1. Interface crack with contact zones. 
where $g(x)$ denotes the gap between the two crack faces. The boundary conditions in the contact zones $-L<x<-a$ and $b<x<L$ are more complicated:

$$
\begin{aligned}
& g(x)=0 \\
& \left|\sigma_{x y}(x, 0)\right|=f\left|\sigma_{y y}(x, 0)\right| \\
& \sigma_{y y}(x, 0) \leq 0 \\
& \operatorname{sgn} \sigma_{x y}(x, 0)=\operatorname{sgn} \frac{d h(x)}{d t}
\end{aligned}
$$

where $f$ is the coefficient of friction, $t$ denotes time and

$$
h(x)=u_{x}^{(2)}(x, 0)-u_{x}^{(1)}(x, 0)
$$

is the tangential shift between the solids. The last expression (7) in the boundary conditions simply reflects the requirement that the sign of the shearing tractions be consistent with the direction of slip at any given instant. We assume that, for loads that start from zero and are increased monotonically, slip takes place over the whole extent of the contact zones and is in the same direction as for the case of no friction. Thus, for $\beta>0, h(x)$ is negative in $-L<x<-a$, positive in $b<x<L$ and changes monotonically with time as the level of loading is increased. Consequently, (5) can be replaced with

$$
\begin{array}{lc}
\sigma_{x y}(x, 0)=f \sigma_{y y}(x, 0) ; & -L<x<-a \\
\sigma_{x y}(x, 0)=-f \sigma_{y y}(x, 0) ; & b<x<L
\end{array}
$$

and (7) discarded. Our formulation automatically incorporates continuity of displacements outside the crack $(|x|>L)$ and continuity of tractions everywhere along the interface $(|x|<\infty)$, and we refrain from writing these conditions.

It is convenient to formulate the problem by considering the crack as an array of distributed edge dislocations. We recall for this purpose the following results for a discrete interface dislocation [8]: Suppose that a discrete edge dislocation is placed at the point $(\xi, 0)$ of the interface (see Fig. 1 for the orientation of the coordinate axes with respect to the two solids). An edge dislocation with a Burgers vector in the $x$-direction (interface glide dislocation) induces the following traction components at the interface

$$
\sigma_{x y}(x, 0 ; \xi)=-\frac{C b_{x}}{\pi} \frac{1}{\xi-x} ; \quad \sigma_{y y}(x, 0 ; \xi)=-\beta C b_{x} \delta(\xi-x)
$$

If the Burgers vector is in the $y$-direction (interface climb dislocation), the traction components are

$$
\sigma_{x y}(x, 0 ; \xi)=\beta C b_{y} \delta(\xi-x) ; \quad \sigma_{y y}(x, 0 ; \xi)=-\frac{C b_{y}}{\pi} \frac{1}{\xi-x}
$$

where $\delta$ denotes the Dirac delta function, and $b_{x}$ and $b_{y}$ are the components of the Burgers vector. Moreover,

$$
C=\frac{2 \mu_{1}(1+\alpha)}{\left(\kappa_{1}+1\right)\left(1-\beta^{2}\right)}=\frac{2 \mu_{2}(1-\alpha)}{\left(\kappa_{2}+1\right)\left(1-\beta^{2}\right)}
$$


with

$$
\begin{array}{rlrl}
\alpha & =\frac{\mu_{2}\left(\kappa_{1}+1\right)-\mu_{1}\left(\kappa_{2}+1\right)}{\mu_{2}\left(\kappa_{1}+1\right)+\mu_{1}\left(\kappa_{2}+1\right)} ; & -1 \leq \alpha \leq 1 \\
\beta=\frac{\mu_{2}\left(\kappa_{1}-1\right)-\mu_{1}\left(\kappa_{2}-1\right)}{\mu_{2}\left(\kappa_{1}+1\right)+\mu_{1}\left(\kappa_{2}+1\right)} ; & -\frac{1}{2} \leq \beta \leq \frac{1}{2}
\end{array}
$$

where $\kappa=3-4 \nu$ in plane strain.

Suppose that the interface transmitted the shearing tractions $S(x)$ and normal tractions $T(x)$ in the absence of a crack. In order to account for the crack, the dislocation distributions must modify the stress fields so that the boundary and auxiliary conditions written before are satisfied. Using (11-14), the boundary conditions (1), (2), (9) and (10) yield the following integral equations for the densities $B_{x}(x)$ and $B_{y}(x)$ of the glide and climb dislocations:

$$
\begin{aligned}
& S(x)+C\left\{\beta B_{y}(x)-\frac{1}{\pi} \int_{-L}^{L} \frac{B_{x}(\xi)}{\xi-x} d \xi\right\}=0 ; \quad-a<x<b \\
& T(x)-C\left\{\beta B_{x}(x)+\frac{1}{\pi} \int_{-a}^{b} \frac{B_{y}(\xi)}{\xi-x} d \xi\right\}=0 ; \quad-a<x<b \\
& S(x)-\frac{C}{\pi} \int_{-L}^{L} \frac{B_{x}(\xi)}{\xi-x} d \xi=-f \operatorname{sgn} x\left\{T(x)-\beta C B_{x}(x)-\frac{C}{\pi} \int_{-a}^{b} \frac{B_{y}(\xi)}{\xi-x} d \xi\right\} ; \\
& -L<x<-a \text { and } b<x<L
\end{aligned}
$$

The dislocation densities are simply

$$
B_{x}(x)=-\frac{d h(x)}{d x} ; \quad B_{y}(x)=-\frac{d g(x)}{d x}
$$

and we note that $B_{y}(x)$ vanishes in the contact zones $-L<x<-a$ and $b<x<L$. The last integral equation (20) incorporates both (9) and (10) by using the signum function. In writing (20), it has been assumed that the right contact zone does not extend to the left of the origin, or that $b>0$.

In order to ensure single valued displacements, or no net dislocation, we must also require that

$$
\int_{-L}^{L} B_{x}(\xi) d \xi=0 ; \quad \int_{-a}^{b} B_{y}(\xi) d \xi=0
$$

Noting that $B_{y}(x)$ vanishes in the contact zones $-L<x<-a$ and $b<x<L$, and that the brace on the right side of (20) vanishes in the open part of the crack $-a<x<b$ on account of (19), it is clear that (18) and (20) can be combined into a single integral equation valid in the interval $-L<x<L$ :

$$
\begin{aligned}
S(x)+C\left\{\beta B_{y}(x)-\frac{1}{\pi} \int_{-L}^{L} \frac{B_{x}(\xi)}{\xi-x} d \xi\right\}=-f & \operatorname{sgn} x\left\{T(x)-\beta C B_{x}(x)\right. \\
& \left.-\frac{C}{\pi} \int_{-a}^{b} \frac{B_{y}(\xi)}{\xi-x} d \xi\right\} ; \quad-L<x<L
\end{aligned}
$$




\section{Reduction of the integral equations}

The problems of common interest result when we take $S(x)$ and $T(x)$ as constant, since this corresponds to uniform loading at infinity. The shear load is perhaps more challenging, because the problem lacks symmetry, and we consider $S(x)=S=$ const and $T(x)=0$ in the sequel.

The governing integral equations are then

$$
\begin{aligned}
& f \beta \operatorname{sgn} x B_{x}(x)+\frac{1}{\pi} \int_{-L}^{L} \frac{B_{x}(\xi)}{\xi-x} d \xi=\frac{S}{C}+\beta[H(x+a)-H(x-b)] B_{y}(x) \\
&+\frac{f}{\pi} \operatorname{sgn} x \int_{-a}^{b} \frac{B_{y}(\xi)}{\xi-x} d \xi \equiv F(x) ; \quad-L<x<L \\
& \beta B_{x}(x)+\frac{1}{\pi} \int_{-a}^{b} \frac{B_{y}(\xi)}{\xi-x} d \xi=0 ; \quad-a<x<b
\end{aligned}
$$

The Heaviside step functions have been put into (26) for computational convenience in order to automatically incorporate the fact that $B_{y}(x)$ vanishes in the contact zones.

As seen from (22), $B_{y}(x)$ must be continuous in $-a<x<b$. Moreover, $B_{y}(x)$ must be bounded at $-a$ and $b$, because the transition from contact to separation has to be smooth [9]. Similarly, $B_{x}(x)$ must be continuous in $-L<x<L$, but is expected to be singular at $\pm L$.

Next, we view (26) as an integral equation for $B_{x}(x)$ and solve it by formally treating $B_{y}(x)$ as known. Thus, $[10, \S 118]$,

$$
B_{x}(x)=D w(x)+\frac{1}{1+f^{2} \beta^{2}}\left\{f \beta \operatorname{sgn} x F(x)-\frac{w(x)}{\pi} \int_{-L}^{L} \frac{F(\xi) d \xi}{(\xi-x) w(\xi)}\right\} ; \quad-L<x<L
$$

where $F(x)$ denotes the right side of (26),

$$
w(x)=\left(L^{2}-x^{2}\right)^{-B}
$$

is the characteristic function of (26), and

$$
\cot \pi B=f \beta ; \quad 0<B<1
$$

With our assumption about the direction of slip, $B \leq \frac{1}{2}$, and the singularity in $B_{x}(x)$ is weaker than inverse square root. Using the Poincaré-Bertrand transformation formula $[10, \S 23],(28)$ yields

$$
\begin{aligned}
& B_{x}(x)=D w(x)+\frac{1}{1+f^{2} \beta^{2}}\left\{f \operatorname{sgn} x\left(\frac{\beta S}{C}-B_{y}(x)+\beta^{2}[H(x+a)-H(x-b)] B_{y}(x)\right)\right. \\
& -\frac{S}{\pi C} w(x) \int_{-L}^{L} \frac{d \xi}{(\xi-x) w(\xi)}-\frac{f^{2} \beta}{\pi} \int_{-a}^{b} \frac{B_{y}(\xi)}{\xi-x} d \xi-\frac{\beta}{\pi} w(x) \int_{-a}^{b} \frac{B_{y}(\xi) d \xi}{(\xi-x) w(\xi)} \\
& \left.+\frac{f}{\pi^{2}} w(x) \int_{-a}^{b} \frac{B_{y}\left(\xi^{\prime}\right)}{\xi^{\prime}-x} \int_{-L}^{L}\left[\frac{1}{\xi-x}+\frac{1}{\xi^{\prime}-\xi}\right] \frac{\operatorname{sgn} \xi}{w(\xi)} d \xi d \xi^{\prime}\right\} ; \quad-L<x<L
\end{aligned}
$$

The constant $D$ is determined by introducing (30) into (23) and using some known 
integral formulas from Gradshteyn and Ryzhik [11]. Thus,

$$
\begin{aligned}
D\left(1+f^{2} \beta^{2}\right)(2 L)^{1-2 B} \frac{\Gamma(1-B) \Gamma(1-B)}{\Gamma(2-2 B)}=f\left(1-\beta^{2}\right) \int_{-a}^{b} B_{y}(\xi) \operatorname{sgn} \xi d \xi \\
+\frac{\beta}{\pi}\left(1+f^{2}\right)(2 L)^{-2 B} \frac{\Gamma(-B) \Gamma(1-B)}{\Gamma(1-2 B)} \int_{-a}^{b} \frac{B_{y}(\xi)}{w(\xi)} F\left(2 B, 1 ; 1+B ; \frac{L-\xi}{2 L}\right) d \xi \\
+\frac{f L^{2 B}}{\pi^{2} B} \int_{-L}^{L} w(x) \int_{-a}^{b} \frac{B_{y}(\xi)}{\xi-x}\left[F\left(-B, 1 ; 1-B ; \frac{L^{2}-x^{2}}{L^{2}}\right)\right. \\
\left.-F\left(-B, 1 ; 1-B ; \frac{L^{2}-\xi^{2}}{L^{2}}\right)\right] d \xi d x \\
-\frac{S}{\pi C}(2 L)^{2 B} \frac{\Gamma(B) \Gamma(1+B)}{\Gamma(1+2 B)} \int_{-L}^{L} w(x) F\left(-2 B, 1 ; 1-B ; \frac{L-x}{2 L}\right) d x+2 f \beta \frac{S L}{C}
\end{aligned}
$$

where $F(a, b ; c ; z)$ denotes a hypergeometric function.

Substituting $B_{x}(x)$ from (30) into (27) we get

$$
\begin{aligned}
-f \beta & \left(1-\beta^{2}\right) \operatorname{sgn} x B_{y}(x)+\frac{1-\beta^{2}}{\pi} \int_{-a}^{b} \frac{B_{y}(\xi) d \xi}{\xi-x}=\frac{\beta^{2}}{\pi} \int_{-a}^{b} \frac{B_{y}(\xi)}{\xi-x}\left[\frac{w(x)}{w(\xi)}-1\right] d \xi \\
& -\frac{f \beta}{\pi^{2}} w(x) \int_{-a}^{b} \frac{B_{y}\left(\xi^{\prime}\right)}{\xi^{\prime}-x} \int_{-L}^{L}\left[\frac{1}{\xi-x}+\frac{1}{\xi^{\prime}-\xi}\right] \frac{\operatorname{sgn} \xi}{w(\xi)} d \xi d \xi^{\prime} \\
& +\frac{\beta S}{\pi C} w(x) \int_{-L}^{L} \frac{d \xi}{(\xi-x) w(\xi)}-\frac{f \beta^{2} S}{C} \operatorname{sgn} x-D \beta\left(1+f^{2} \beta^{2}\right) w(x) ; \quad-a<x<b
\end{aligned}
$$

which is a singular integral equation for $B_{y}(x)$ containing a Cauchy-type kernel. The dominant part appears on the left side. Equation (32) becomes

$$
\begin{aligned}
-f \beta & \left(1-\beta^{2}\right) \operatorname{sgn} x B_{y}(x)+\frac{1-\beta^{2}}{\pi} \int_{-a}^{b} \frac{B_{y}(\xi)}{\xi-x} d \xi=\frac{\beta^{2}}{\pi} \int_{-a}^{b} \frac{B_{y}(\xi)}{\xi-x}\left[\frac{w(x)}{w(\xi)}-1\right] d \xi \\
& -\frac{f \beta}{\pi^{2}} w(x) \int_{-a}^{b} \frac{B_{y}(\xi)}{\xi-x}\left\{\pi f \beta\left[\frac{1}{w(x)}-\frac{1}{w(\xi)}\right]-\frac{L^{2 B}}{B}\left[F\left(-B, 1 ; 1-B ; \frac{L^{2}-x^{2}}{L^{2}}\right)\right.\right. \\
& \left.\left.-F\left(-B, 1 ; 1-B ; \frac{L^{2}-\xi^{2}}{L^{2}}\right)\right]\right\} d \xi+\frac{\beta S}{\pi C} w(x)\left[\frac{\pi f \beta}{w(x)}-(2 L)^{2 B} \frac{\Gamma(B) \Gamma(1+B)}{\Gamma(1+2 B)}\right. \\
\times & \left.F\left(-2 B, 1 ; 1-B ; \frac{L-x}{2 L}\right)\right]-\frac{f \beta^{2} S}{C} \operatorname{sgn} x-D \beta\left(1+f^{2} \beta^{2}\right) w(x) ; \quad-a<x<b
\end{aligned}
$$

after the evaluation of some of the integrals.

In the next steps, the following substitutions are used whenever they improve the convergence of the hypergeometric series without introducing non-integrable singularities:

$$
\begin{aligned}
F(2 B, 1 ; 1+B ; z) & =-\frac{B}{1-2 B} \frac{1}{1-z}+\frac{1-B}{1-2 B} \frac{1}{1-z} F(2 B-1,1 ; 1+B ; z) \\
F(-2 B, 1 ; 1-B ; z) & =\frac{1}{1-z}-\frac{1+B}{1-B} \frac{z}{1-z} F(-2 B, 1 ; 2-B ; z)
\end{aligned}
$$




$$
\begin{array}{r}
F(-B, 1 ; 1-B ; z)=\frac{B}{1+B} \frac{1}{1-z}+\frac{1}{1+B} \frac{1}{1-z} F(-B-1,1 ; 1-B ; z) \\
F\left(-B, 1 ; 1-B ; \frac{L^{2}-x^{2}}{L^{2}}\right)=\frac{1}{1+B} \frac{L^{2}}{x^{2}} \sum_{n=0}^{\infty}\left\{\frac{(-B-1)_{n}}{(1-B)_{n}}\left[\left(1-\frac{x^{2}}{L^{2}}\right)^{n}-1\right]\right\}
\end{array}
$$

where

$$
(A)_{n}=A(A+1) \cdots(A+n-1) ; \quad(A)_{0}=1
$$

Equations (31) and (33) are normalized by the change of variables

$$
\begin{array}{ll}
\frac{x}{L}=\delta \zeta+\sigma ; & \frac{\xi}{L}=\delta \omega+\sigma \\
\delta=\frac{b+a}{2 L} ; & \sigma=\frac{b-a}{2 L}
\end{array}
$$

Thus (33) and (31) become

$$
\begin{aligned}
-f \beta & \left(1-\beta^{2}\right) \operatorname{sgn}(\delta \zeta+\sigma) B_{y}(\zeta)+\frac{1-\beta^{2}}{\pi} \int_{-1}^{1} \frac{B_{y}(\omega)}{\omega-\zeta} d \omega \\
= & \frac{\beta^{2}\left(1+f^{2}\right)}{\pi} \int_{-1}^{1} \frac{B_{y}(\omega)}{\omega-\zeta}\left[\left(\frac{1-(\delta \omega+\sigma)^{2}}{1-(\delta \zeta+\sigma)^{2}}\right)^{B}-1\right] d \omega \\
& +\frac{f \beta}{\pi^{2} B(1+B)}\left[1-(\delta \zeta+\sigma)^{2}\right]^{-B} \int_{-1}^{1} \frac{B_{y}(\omega)}{\omega-\zeta} \\
& \times\left\{\frac{1}{(\delta \zeta+\sigma)^{2}} \sum_{n=0}^{\infty} \frac{(-B-1)_{n}}{(1-B)_{n}}\left[\left(1-(\delta \zeta+\sigma)^{2}\right)^{n}-1\right]\right. \\
& \left.-\frac{1}{(\delta \omega+\sigma)^{2}} \sum_{n=0}^{\infty} \frac{(-B-1)_{n}}{(1-B)_{n}}\left[\left(1-(\delta \omega+\sigma)^{2}\right)^{n}-1\right]\right\} d \omega \\
& -\frac{\beta S}{\pi C} \frac{2^{2 B} \Gamma(B) \Gamma(1+B)}{\Gamma(1+2 B)(1+\delta \zeta+\sigma)}\left[1-(\delta \zeta+\sigma)^{2}\right]^{-B}\left\{2-\frac{1+B}{1-B}(1-\delta \zeta-\sigma)\right. \\
& \left.\times F\left(-2 B, 1 ; 2-B ; \frac{1-\delta \zeta-\sigma}{2}\right)\right\}+\frac{f \beta^{2} S}{C}[1-\operatorname{sgn}(\delta \zeta+\sigma)] \\
- & D \beta\left(1+f^{2} \beta^{2}\right) L^{-2 B}\left[1-(\delta \zeta+\sigma)^{2}\right]^{-B} ; \\
D(1+ & \left.f^{2} \beta^{2}\right) L^{-2 B} \frac{2^{1-2 B} \Gamma(1-B) \Gamma(1-B)}{\delta \Gamma(2-2 B)} \\
= & f\left(1-\beta^{2}\right) \int_{-1}^{1} B_{y}(\omega) \operatorname{sgn}(\delta \omega+\sigma) d \omega \\
& +\frac{\beta}{\pi}\left(1+f^{2}\right) 2^{1-2 B} \frac{\Gamma(1-B) \Gamma(1-B)}{\Gamma(2-2 B)} \int_{-1}^{1} B_{y}(\omega) \frac{(1-\delta \omega-\sigma)^{B}}{(1+\delta \omega+\sigma)^{1-B}} d \omega \\
& -\frac{\beta}{\pi}\left(1+f^{2}\right) 2^{1-2 B} \frac{\Gamma(1-B) \Gamma(2-B)}{B \Gamma(2-2 B)} \int_{-1}^{1} B_{y}(\omega) \frac{(1-\delta \omega-\sigma)^{B}}{(1+\delta \omega+\sigma)^{1-B}} \\
& \left.\times F\left(2 B-1,1 ; 1+B ; \frac{1-\delta \omega-\sigma}{2}\right)\right) d \omega \\
& (2+\delta
\end{aligned}
$$




$$
\begin{aligned}
& +\frac{f}{\pi^{2} B(1+B)} \int_{-1}^{1}\left(1-s^{2}\right)^{-B} \int_{-1}^{1} \frac{B_{y}(\omega)}{\delta \omega+\sigma-s}\left\{\frac{1}{s^{2}} \sum_{n=0}^{\infty} \frac{(-B-1)_{n}}{(1-B)_{n}}\left[\left(1-s^{2}\right)^{n}-1\right]\right. \\
& \left.-\frac{1}{(\delta \omega+\sigma)^{2}} \sum_{n=0}^{\infty} \frac{(-B-1)_{n}}{(1-B)_{n}}\left[\left(1-(\delta \omega+\sigma)^{2}\right)^{n}-1\right]\right\} d \omega d s \\
& -\frac{S}{\pi C \delta} \frac{2^{2 B} \Gamma(B) \Gamma(1+B)}{\Gamma(1+2 B)} \int_{-1}^{1}\left(1-s^{2}\right)^{-B} \\
& \times F\left(-2 B, 1 ; 1-B ; \frac{1-s}{2}\right) d s+\frac{2 f \beta S}{C \delta}
\end{aligned}
$$

The last two equations together with (24) are the relations necessary for the determination of $B_{y}(x)$. It may be noted that all integrals in (42) containing $B_{y}(x)$ involve bounded kernels and, consequently, the constant $D$ contributes, as it should, only to the regular part of the integral equation when substituted into (41). Once $B_{y}(x)$ has been found, the other dislocation density $B_{x}(x)$ can be computed from (30).

The shearing stresses ahead of the crack tips are on basis of (11) and (13)

$$
\sigma_{x y}(x, 0)=S-\frac{C}{\pi} \int_{-L}^{L} \frac{B_{x}(\xi)}{\xi-x} d \xi ; \quad|x|>L
$$

The stress intensity factors in shear can be defined as

$$
\begin{aligned}
K_{2}\left( \pm L^{+}\right) & =\lim _{x \rightarrow \pm L}\left\{[2( \pm x-L)]^{B} \sigma_{x y}(x, 0)\right\} \\
& = \pm C L^{-B} \lim _{x \rightarrow \pm L}\left[\left(L^{2}-x^{2}\right)^{B} B_{x}(x)\right]
\end{aligned}
$$

while the stress intensity factors connected with the normal tractions in the contact zones are [4]

$$
K_{1}\left( \pm L^{-}\right)=\mp \beta K_{2}\left( \pm L^{+}\right)
$$

The normal traction in the larger of the contact zones $b<x<L$ follows from (12) and (14)

$$
\sigma_{y y}(x, 0)=-C\left\{\beta B_{x}(x)+\frac{1}{\pi} \int_{-a}^{b} \frac{B_{y}(\xi)}{\xi-x} d \xi\right\}
$$

\section{Numerical solution}

For $S>0$ and $\beta>0$, the left contact zone is expected to be very small and the slope of the gap very large at $x=-a$ [5]. This situation is best approximated in the numerical solution by taking $B_{y}(x)$ as singular at $x=-a$ and then driving the intensity of the singularity to zero by adjusting $a$. Therefore, the density of the climb dislocations is expressed as

$$
\dot{B}_{\mathrm{y}}(\omega)=\frac{S}{C}(1+\omega)^{\mathrm{B}-1}(1-\omega)^{\mathrm{B}} \phi(\omega)
$$

where $\phi(\omega)$ is a regular function and $B$ is given by (29). The integral equation (41) together with (42) can be discretized using the scheme developed by Krenk [12]. 
Accordingly, the dominant part of (41) becomes

$$
-f \beta\left(1-\beta^{2}\right) \operatorname{sgn}(\delta \zeta+\sigma) B_{y}(\zeta)+\frac{1-\beta^{2}}{\pi} \int_{-1}^{1} \frac{B_{y}(\omega)}{\omega-\zeta} d \omega=\frac{\left(1-\beta^{2}\right) S}{\pi C} \sum_{i=1}^{n} \frac{A_{i}^{(n)} \phi\left(\omega_{i}\right)}{\omega_{i}-\zeta_{k}}
$$

where $\omega_{i}$ and $\zeta_{k}$ are the roots of the Jacobi polynomials

$$
\begin{array}{rlrl}
P_{n}^{(B, B-1)}\left(\omega_{i}\right) & =0 ; & i & =1,2, \ldots, n \\
P_{n}^{(-B, 1-B)}\left(\zeta_{k}\right) & =0 ; \quad k & =1,2, \ldots, n
\end{array}
$$

and $A_{i}^{(n)}$ are the coefficients of the Gauss-Jacobi quadrature formula with the weight function $(1+\omega)^{B-1}(1-\omega)^{B}$. Equations (24) and (42), and the remaining terms are also discretized by using the Gauss-Jacobi quadrature, but the resulting expressions are too lengthy to record.

It was found in the course of the numerical solution that, for $\beta>0$, the parameter $a$ defining the extent of the left contact zone depends almost exclusively on the local value of $B_{y}(x)$. For $\beta=0.5, a / L$ falls between $1-10^{-6}$ and $1-10^{-7}$. No effort was made to determine $a / L$ more accurately because it does not affect appreciably the stresses except at $x=-a$.

The dimensionless shear stress intensity factors $\hat{K}_{2}( \pm L)=K_{2}( \pm L)\left(1+f^{2} \beta^{2}\right) / S L^{B}$ are given in Table 1 for a few values of the friction coefficient. The discrepancy in $\hat{K}_{2}(-L)$ for $f=0$ between the present result and that from [5] is probably due to the slow convergence of some of the hypergeometric functions at $x=-a$. The extent of the large contact zone on the right is shown in Figure 2. The gap between the two solids and the contact tractions in $b<x<L$ are given for $f=0$ and 1.0 , and $\beta=0.5$ in Figures 3 and 4.

TABLE 1

\begin{tabular}{lll}
\hline$f$ & $\hat{K}_{2}(+L)$ & $\hat{K}_{2}(-L)$ \\
\hline 1.0 & 1.08 & -9.55 \\
0.7 & 1.00 & -4.27 \\
0.5 & 0.96 & -2.27 \\
0.3 & 0.95 & -1.12 \\
0 & 1.03 & -0.40 \\
0 from $[5]$ & 1.03 & -0.45 \\
\hline
\end{tabular}

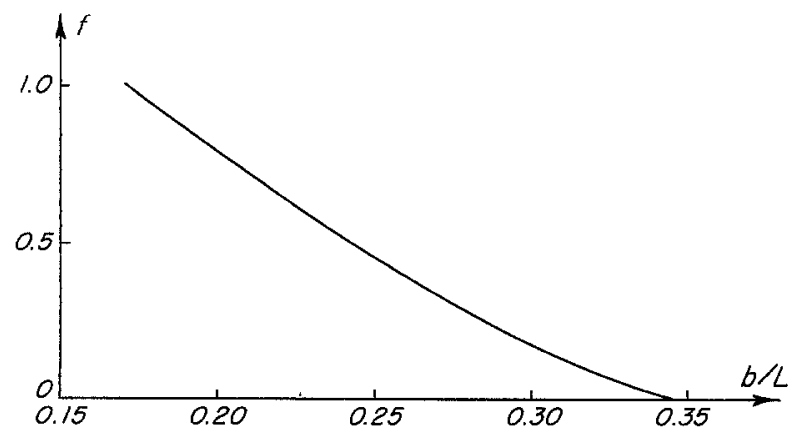

Figure 2. Effect of friction on the extent of the large contact zone for shear loading. 


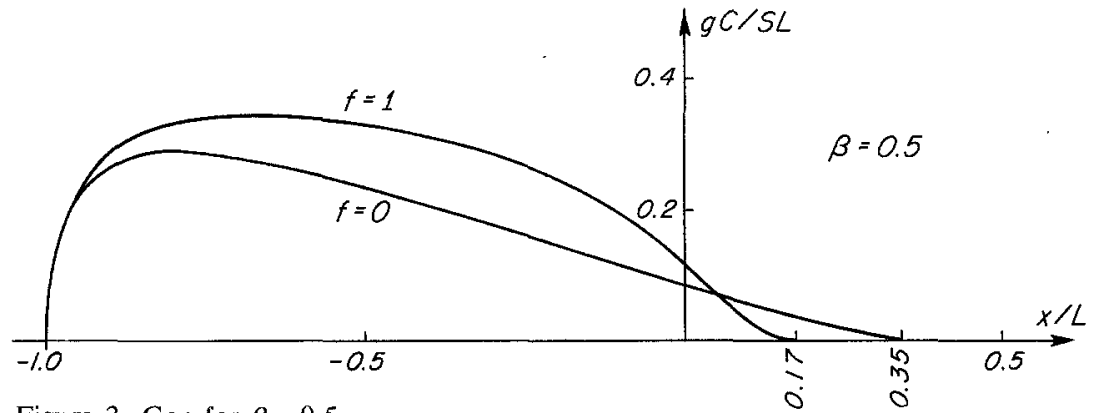

Figure 3. Gap for $\beta=0.5$.

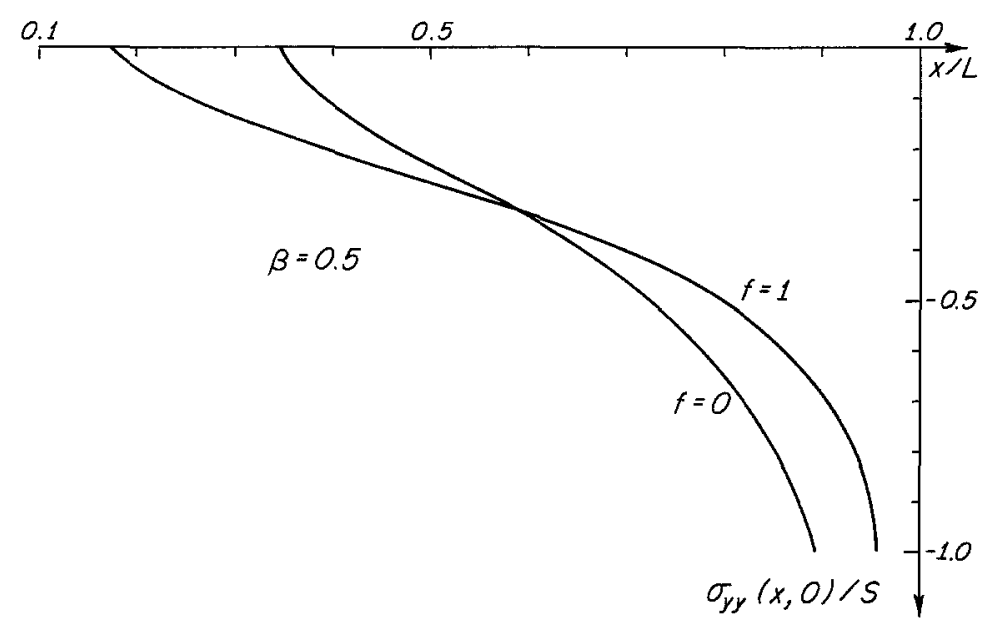

Figure 4. Normal tractions in the large contact zone for $\beta=0.5$.

\section{Acknowledgment}

The work of one of the authors (M. C.) was supported by the National Science Foundation through the Grant ENG77-25032.

\section{REFERENCES}

[1] A. H. England, Journal of Applied Mechanics, 32 (1965) 400.

[2] B. M. Malyshev and R. L. Salganik, International Journal of Fracture Mechanics, 1 (1965) 114.

[3] M. Comninou, Journal of Applied Mechanics, 44 (1977) 631.

[4] M. Comninou, Journal of Applied Mechanics, 44 (1977) 780.

[5] M. Comninou, Journal of Applied Mechanics, 45 (1978) 287.

[6] L. E. Goodman, Journal of Applied Mechanics, 29 (1962) 515.

[7] J. Dundurs and M. Comninou, (unpublished work).

[8] M. Comninou, Philosophical Magazine, 36 (1977) 1281.

[9] J. Dundurs and M. Comninou, Journal of Elasticity, 9 (1979) 71.

[10] N. I. Muskhelishvili, Singular Integral Equations, Noordhoff (1953).

[11] I. S. Gradshteyn and I. W. Ryzhik, Tables of Integrals, Series and Products, Academic Press (1965).

[12] S. Krenk, Quarterly of Applied Mathematics, 33 (1975) 225. 